\title{
Effect of nurse-directed hypertension treatment among First Nations people with existing hypertension and diabetes mellitus: the Diabetes Risk Evaluation and Microalbuminuria (DREAM 3) randomized controlled trial
}

\author{
Sheldon W. Tobe, George Pylypchuk, Joan Wentworth, Alexander Kiss, John Paul Szalai, \\ Nancy Perkins, Susan Hartman, Laurie Ironstand, Jacqueline Hoppe
}

Published at www.cmaj.ca on Apr. 4, 2006. An abridged version of this article appeared in the Apr. 25, 2006, issue of CMAJ.

\section{ABSTRACT}

Background: First Nations people with diabetes mellitus and hypertension are at greater risk of renal and cardiovascular complications than are non-native patients because of barriers to health care services. We conducted this randomized controlled trial to assess whether a community-based treatment strategy implemented by home care nurses would be effective in controlling hypertension in First Nations people with existing hypertension and type 2 diabetes.

Methods: We compared 2 community-based strategies for controlling hypertension in First Nations people with existing hypertension and diabetes. In the intervention group, a home care nurse followed a predefined treatment algorithm of pharmacologic antihypertensive therapy. In the control group, treatment decisions were made by each subject's primary care physician. The primary outcome measure was the difference between the 2 groups in the change in systolic blood pressure after 12 months. Secondary outcome measures were the change in diastolic blood pressure over time, the change in urine albumin status and the incidence of adverse events.

Results: Both groups experienced a significant reduction in systolic blood pressure by the final visit (by 24.0 [standard deviation (SD) 13.5 ] $\mathrm{mm} \mathrm{Hg}$ in the intervention group and by 17.0 [SD 18.6] $\mathrm{mm} \mathrm{Hg}$ in the control group); $p<0.001$ in each case). However, the difference between the 2 groups in this change was not significant. Patients in the intervention group had a larger decrease in diastolic blood pressure over time than did those in the control group (by 11.6 [SD 10.6] $\mathrm{mm} \mathrm{Hg} \mathrm{v.} 6.8$ [SD 11.1] $\mathrm{mm} \mathrm{Hg}$ respectively; $p=0.05$ ). The groups did not differ significantly in terms of changes in urine albumin excretion or incidence of adverse events.

Interpretation: High rates of blood pressure control in the community were achieved in both groups in the DREAM 3 study. The addition of a home care nurse to implement a treatment strategy for blood pressure control was more ef- fective in lowering diastolic than systolic blood pressure compared with home care visits for blood pressure monitoring alone and follow-up treatment by a family physician.

Cite this article as $C M A J$ 2006;174(9). DOI:I0.1503/cmaj.050030

$\mathrm{P}$ atients with type 2 diabetes mellitus and hypertension are at increased risk of cardiovascular events and chronic renal failure requiring dialysis. ${ }^{1}$ In Canada, the risk of progression to end-stage renal disease among such people is 7 times higher in the First Nations population than in the non-native population. ${ }^{2}$ Although effective strategies exist for treating hypertension, in First Nations populations treatment of hypertension is challenging because of reduced access to ambulatory care and a hesitancy regarding modern disease management strategies. ${ }^{3}$ Clinical studies in hypertension have shown that high rates of blood pressure control are attainable; ${ }^{4}$ in the research setting, nurse-implemented algorithms supervised by a physician have been found to contribute to efficacious blood pressure control. ${ }^{5}$

In 1998 the Diabetes Risk Evaluation and Microalbuminuria (DREAM 1) study was initiated at the request of the Battlefords Tribal Council Indian Health Services to assess the prevalence of risk factors for end-stage renal disease (ESRD) among First Nations people with type 2 diabetes in the Battlefords region of northern Saskatchewan. ${ }^{6}$ In 2000 the DREAM 2 pilot study demonstrated a reduction of systolic blood pressure when home care nurses measured blood pressure at regular intervals and notified the patient's family physician. ${ }^{6}$ In this article, we report the findings of the DREAM 3 study, which was designed to evaluate the effect on blood pressure control of an algorithm of pharmacologic therapy implemented by home care nurses in a community setting.

\section{Methods}

This was a randomized, prospective, open-label study with 2 parallel groups. We recruited First Nations people registered 
with the Battlefords Tribal Council Indian Health Services through community screening clinics and during visits by home care nurses and home health aides between September $200 \mathrm{I}$ and March 2003. Inclusion and exclusion criteria are found in Box I. All patients provided written informed consent, and an interpreter was available at all stages of the study. The protocol was in accordance with the Declaration of Helsinki and was approved by the ethics committees of the Sunnybrook and Women's College Health Sciences Centre, the University of Toronto and the University of Saskatchewan.

All participants were seen by a home care nurse in the medical clinic on their reserve at baseline, at 6 weeks and at 3 , 6, 9 and 12 months after enrolment. They were all seen by a hypertension specialist (S.W.T. or G.P.) at their baseline and final visits. Participants were accompanied by a home health aide from their reserve, when possible, to help with language and cultural issues. All patients received healthy lifestyle classes, focusing on a healthier dietary regimen, exercise (30 minutes per day, 5 days per week), smoking cessation and drug adherence. ${ }^{7}$ Participants were instructed to take their morning medications before each home care visit. Following

Box 1: Inclusion and exclusion criteria for the DREAM 3 study

Inclusion criteria

- Age $\geq 18$ yr

- Type 2 diabetes mellitus

- Persistent hypertension (systolic pressure $\geq 130 \mathrm{~mm} \mathrm{Hg}$, diastolic pressure $\geq 80 \mathrm{~mm} \mathrm{Hg}$, or both)

\section{Exclusion criteria}

- Use of $\beta$-blocker (subjects randomly assigned to the intervention group had their antihypertensive therapy switched at baseline, with no time to taper $\beta$-blocker therapy)

- Woman of child-bearing age not able to use a reliable method of birth control

- Connective tissue disorder

- Severe systemic or malignant disease

- Inability to follow the protocol

- Bilateral renal artery stenosis and other causes of secondary hypertension

- Serum creatinine level $>250 \mu \mathrm{mol} / \mathrm{L}$

- Cerebrovascular event within 6 mo before study recruitment

- Valvular heart disease

- Unstable angina

- Myocardial infarction

- Revascularization procedure within 3 mo before study recruitment

- Heart failure (NYHA class III or IV)

- Cardiac arrhythmia requiring medical treatment or heart block

- Active hepatic disease

Note: DREAM = Diabetes Risk Evaluation and Microalbuminuria, NYHA $=$ New York Heart Association. each visit, a letter summarizing the patient's current medications, blood pressure and updated laboratory test results was completed and forwarded to the primary care physician. A summary of the clinical practice guidelines for managing hypertension in people with diabetes ${ }^{8}$ was included with each letter. Also included were algorithms for glucose and lipid control (see online appendices, available at www.cmaj.ca/cgi /content/full/cmaj.050030/DCI); these algorithms were developed from current clinical practice guidelines. ${ }^{8-10}$

Randomization was performed by means of opaque sealed envelopes using a permuted block design, stratified by the 7 reserves. Envelopes were opened by the home care nurse in the presence of the physician and patient at the end of the baseline visit. Fifty subjects were randomly assigned to the treatment strategy and 49 to the control strategy.

Subjects assigned to the intervention group were asked to discontinue their antihypertensive medication at the baseline visit and to start taking the angiotensin II receptor blocker irbesartan at a dose of $150 \mathrm{mg} / \mathrm{d}$. If on subsequent visits their blood pressure was not controlled, a stepped protocol approach to lower the blood pressure to the target $(<\mathrm{I} 30 / 80$ $\mathrm{mm} \mathrm{Hg}$ ) was begun, administered by the home care nurse under the indirect supervision of the hypertension specialist. The patient's drug regimen was titrated according to the hypertension management algorithm (Fig. I). All medications were offered by the First Nations Drug Benefits Plan. Local pharmacies were asked to provide medications in blister packs to aid adherence. After each medication change, follow-up was arranged with the patient's primary care physician. An accelerated titration schedule was allowed after consultation with the hypertension specialist for patients already taking 2 or more antihypertensive agents at baseline.

Participants assigned to the control group were assessed by the home care nurse on the same schedule as that set out for the intervention group. If their blood pressure was above the target on any study visit, follow-up was arranged with their primary care physician and a letter sent as described earlier.

In all cases, blood pressure was measured on each visit by the home care nurse using the BpTRU automated oscillometric blood pressure cuff (VSM Medtech Ltd., Vancouver). ${ }^{11}$ Blood pressure measurements were obtained at least half an hour after coffee or cigarettes, with the patient seated for 30 minutes; patients did not have to fast. In addition, a random urine sample was taken at baseline and every 3 months to determine the urine albumin:creatinine ratio; all blood and urine samples were sent to the laboratory in North Battleford. Microalbuminuria was defined as a urine albumin:creatinine ratio greater than $2.8 \mathrm{mg} / \mathrm{mmol}$ for women and greater than $2.0 \mathrm{mg} / \mathrm{mmol}$ for men. ${ }^{12}$ Overt nephropathy was defined as a urine albumin:creatinine ratio greater than $28 \mathrm{mg} / \mathrm{mmol}$ for women and greater than $20 \mathrm{mg} / \mathrm{mmol}$ for men. ${ }^{12}$ Renal function and serum potassium tests were repeated no more than 2 weeks after the baseline visit for patients in the treatment group because their drug therapy had been switched to a blocker of the renin-angiotensin aldosterone system.

The primary outcome measure was the mean change in systolic blood pressure from baseline to the final visit in the intervention group compared with the control group. Sec- 
ondary outcome measures included the mean change in diastolic blood pressure over time in the 2 groups, the change in urine albumin status (normal to microalbuminuria to overt nephropathy or back) and the incidence of adverse events.

Estimates of sample size were based on an expected treatment effect of a drop in systolic blood pressure of $20 \mathrm{~mm} \mathrm{Hg}$ in the intervention group (as found by Hoy and colleagues with a similar intervention ${ }^{13}$ ) and a drop of $8 \mathrm{~mm} \mathrm{Hg}$ in the control group (as found in the DREAM 2 study $^{6}$ ). A conservative common standard deviation of $25 \mathrm{~mm} \mathrm{Hg}$ for systolic blood pressure was based on the results of the DREAM 2 study with a conservative correlation of 0.43 between systolic blood pressure readings taken I year apart. With $\alpha$ set at 0.05 , we estimated that 60 subjects per group would provide a repeated-measures of analysis of variance (RANOVA) with $80 \%$ power to detect a significant difference in blood pressure between the 2 groups over time. ${ }^{14}$ The change in blood pressure was determined by a comparison of readings taken at the baseline visit with those taken at the final visit.

Analyses were conducted on an intention-to-treat basis. Early in the study, 2 subjects withdrew before their second visit, specifying that they also withdrew their consent to allow their data to be used for analysis; therefore, these subjects were not included in the intention-to-treat analysis. Following this, subjects were notified during the consent process that their data would be used up to the point of withdrawing consent or to the end of the study. There were no crossovers from the intervention to the control strategy. We used the $t$ test to compare baseline characteristics between groups and to compare measures from baseline to the final visit within groups; we used the $\chi^{2}$ test to compare adequate blood pressure control between groups.

\section{Results}

First Nations people from the Battlefords region at increased risk of diabetes or with known diabetes were screened. A total of 179 were found to have either diabetes or, on further testing, a fasting blood glucose level in the diabetes range (Fig. 2). Of these, $6 \mathrm{I}$ had normal or controlled blood pressure $(<\mathrm{I} 30 / 80 \mathrm{~mm}$ $\mathrm{Hg}$ ), and the rest were invited to participate in the study. Seventeen people declined to participate for various reasons, including travel commitments and a fear of needles. Two other patients were excluded, one because of unstable retinopathy that required therapy and the other because she had become pregnant between the screening and baseline visits.

Data from the final I2-month visit, or from the last available visit for those lost to follow-up, were used in the intention-to-treat analysis. Four patients ( 2 from each group) were not included in the analysis: 2 (one from each group) were withdrawn from the study (one was found to have bilateral renal artery stenosis, an ex-

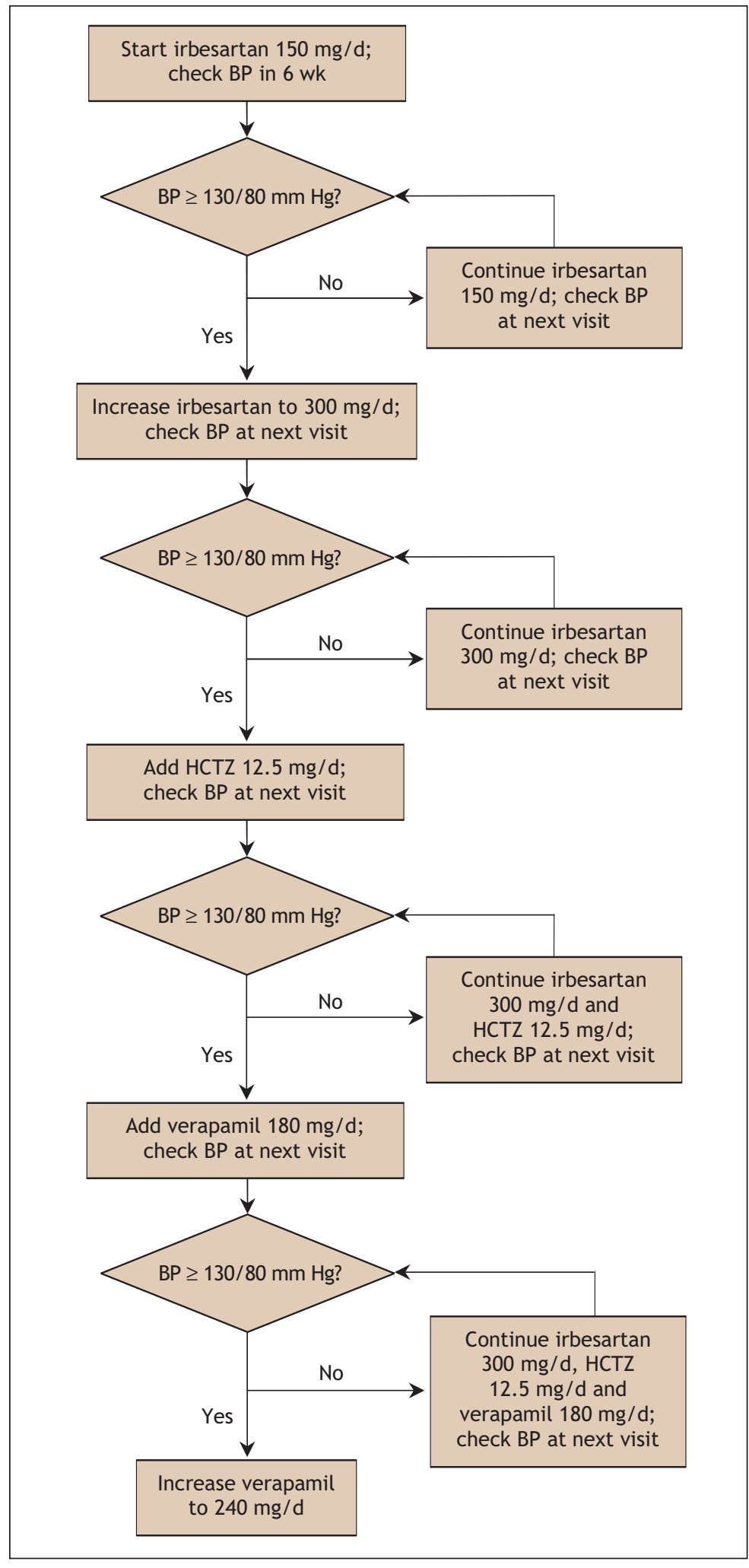

Fig. 1: Hypertension management algorithm followed by home care nurses for patients assigned to the intervention group. Patients were given irbesartan at a starting dose of $150 \mathrm{mg} / \mathrm{d}$. During subsequent visits, if the blood pressure (BP) was greater than the target level, the regimen was stepped up according to the protocol. Verapamil was prescribed in a longacting format. Note: HCTZ = hydrochlorothiazide. 
clusion criteria, and was removed from the study for treatment; the other was enrolled into another study by the patient's family physician), and 2 (one from each group) withdrew their consent to allow use of their data for analysis. Three patients in the intervention group withdrew (one at 6 weeks and 2 at 9 months), and 5 in the control group withdrew (one after the baseline visit, one at 6 weeks, one at 3 months, one at 6 months and one at 9 months); their data were included in the intention-to-treat analysis. Therefore, 95 participants were included in the analysis: 48 in the intervention group and 47 in the control group. There were no significant differences between the 2 groups in demographic and clinical characteristics at baseline (Table I) or at the final visit (data not shown).

In both the intervention and control groups, the change in blood pressure from baseline readings to those at the final visit was statistically significant (Table 2). The reduction in systolic blood pressure was $7.0 \mathrm{~mm} \mathrm{Hg}$ greater in the intervention group than in the control group, but this difference was not statistically significance $(p=0.14)$. The drop in diastolic blood pressure over time was $4.8 \mathrm{~mm} \mathrm{Hg}$ greater in the intervention group than in the control group ( $p=0.05$ ); the RANOVA analysis was repeated 1000 times using bootstrap methods and yielded a median $p$ value of 0.042 . Overall, blood pressure targets (systolic < $130 \mathrm{~mm} \mathrm{Hg}$ and diastolic $<80 \mathrm{~mm} \mathrm{Hg}$ ) were achieved in $48 \%$ of the participants with no significant difference between the 2 groups. Most of the patients in the control group had the doses of their hypertension medications titrated upward during the study by their primary care physicians.

The urine albumin level decreased from baseline to the final visit in both groups, from 157.8 to $115.5 \mathrm{mg} / \mathrm{L}$ in the treatment group and from I64.8 to $124.9 \mathrm{mg} / \mathrm{L}$ in the control group. The differences between and within the 2 groups were not statistically significant (Table 2 ), even when log-transformed levels were analyzed (data not shown). Over the course of the study, the patients who had existing microalbuminuria did not have progression to overt nephropathy (Table 3). In many cases of existing overt nephropathy, the condition regressed to microalbuminuria during the study, although a regression to normal urine albumin levels did not occur (Table 3).

\section{Adverse events}

The incidence of adverse events did not differ significantly between the 2 groups. Ten patients in the intervention group and 7 in the control group required admission to hospital. Of those in the intervention group, 2 were admitted because of myocardial infarction and angina, 1 patient was admitted on 6 occasions (once for management of diabetes and 5 times for treatment of skin infections and a lower-leg amputation), 2 were admitted because of pneumonia, 1 for management of diabetes, 1 because of bleeding hemorrhoids, 1 for treatment of depression, 1 because of a nonspecific allergic reaction (did not require a change in medications) and 1 because of pyelonephritis. In the control group, 2 of the 7 patients were admitted because of myocardial infarction and angina, 1 was admitted because of stroke. The other 4 patients were admitted for cholecystectomy, diabetes management, treatment of pneumonia (admitted twice) and management of resistant hypertension. All I7 patients were assessed at the I2-month visit and were included in the intention-to-treat analysis.

\section{Interpretation}

We found that, among First Nations people with existing hypertension and diabetes enrolled in the DREAM 3 study, the intervention of having a home care nurse monitor their blood

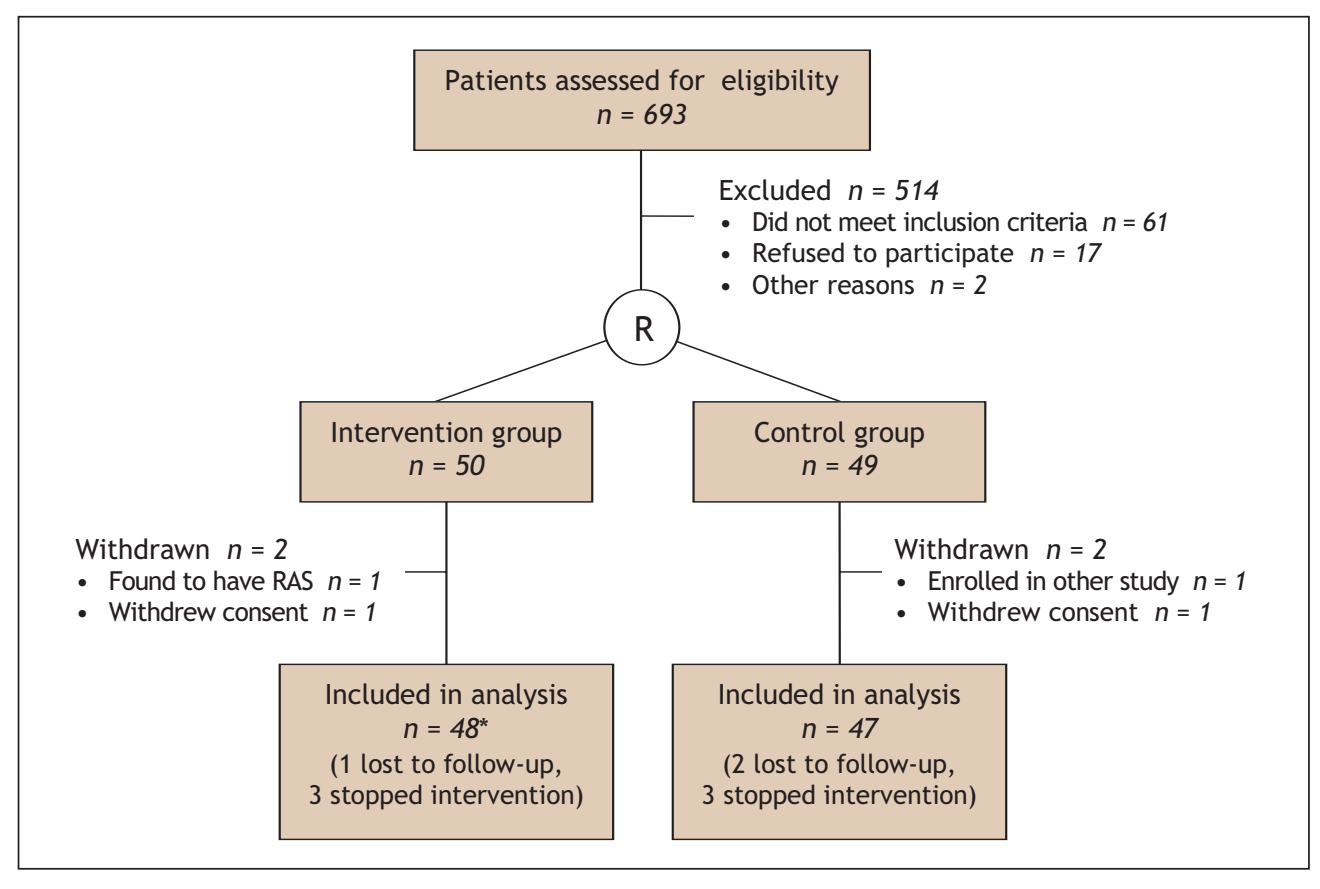

Fig. 2: Flow of patients through the study. $\mathrm{R}=$ randomization, $\mathrm{RAS}=$ renal artery stenosis. 
pressure and follow a predefined treatment algorithm was as effective in lowering systolic blood pressure over time as was the control strategy of having a home care nurse monitor their blood pressure and their family physician decide on follow-up treatment. The intervention was, however, significantly more effective than the control strategy in lowering diastolic pressure. Improvement in blood pressure in both groups over the study period may have been due in part to the patients' involvement in the study program, with education given and blood pressure measured by the home care nurse in both groups. The urine albumin levels fell in both groups, but the difference between the groups was not significant.

Reducing blood pressure to the lower end of the renal autoregulatory range $(<\mathrm{I} 30 / 80 \mathrm{~mm} \mathrm{Hg})$ has been found to result in significant renal protection..$^{15}$ Achieving this target in people with diabetes is currently suboptimal (about $9 \%$ in Canada). ${ }^{16}$ High rates of control of multiple cardiovascular risk factors in the research setting have been achieved with a multidisciplinary approach. ${ }^{17}$ Furthermore, blood pressure control has been found to be cost-effective. ${ }^{18}$ The challenge lies in achieving effective blood pressure control in the community, as has been attempted in Australia. ${ }^{19}$ Specially trained nurses instructed to follow detailed protocols and algorithms under the supervision of a specialist has been shown to improve diabetes outcomes, ${ }^{20}$ as have nurse-led clinics. ${ }^{21} \mathrm{~A}$ Canadian outreach program was effective in lowering blood pressure among people with diabetes living in a rural setting. ${ }^{22}$ New strategies and partnerships that include family physicians, nurses and specialists are required if cardiovascular risk factors such as hypertension are to be controlled.

Our study had several limitations. The home care nurse may have had an effect on blood pressure control independent of the treatment algorithm, which may explain the change

Table 1: Baseline demographic and clinical characteristics and biochemical status of patients in the DREAM 3 study

\begin{tabular}{|lcc}
\hline & \multicolumn{2}{c}{ Group; mean (SD) } \\
\cline { 2 - 3 } Variable & $\begin{array}{c}\text { Intervention } \\
n=50\end{array}$ & $\begin{array}{c}\text { Control } \\
n=49\end{array}$ \\
\hline Age, yr & $55.4(12.9)$ & $55.9(11.5)$ \\
\hline Sex, no. (\%) male & $19(38)$ & $19(39)$ \\
\hline BMl, kg/m ${ }^{2}$ & $34.2(2.6)$ & $33.6(2.1)$ \\
\hline Hip circumference, cm & $112.8(15.0)$ & $111.6(11.1)$ \\
\hline Waist circumference, cm & $111.4(15.5)$ & $111.0(12.1)$ \\
\hline Serum creatinine, $\mu \mathrm{mol} / \mathrm{L}$ & $69.8(17.1)$ & $72.6(16.0)$ \\
\hline Serum potassium, mmol/L & $4.1(0.4)$ & $4.1(0.42)$ \\
\hline Total cholesterol, mmol/L & $5.1(0.9)$ & $5.2(1.1)$ \\
\hline HDL cholesterol, mmol/L & $1.2(0.4)$ & $1.0(0.3)$ \\
\hline Hemoglobin $\mathrm{A}_{1 \mathrm{c}}, \%$ & $7.9(1.9)$ & $7.7(1.8)$ \\
\hline Plasma glucose, mmol/L & $9.7(4.7)$ & $10.1(5.3)$ \\
\hline Urine albumin:creatinine & $25.1(72.6)$ & $33.3(105.1)$ \\
\hline ratio, mg/mmol & & \\
\hline
\end{tabular}

Note: DREAM = Diabetes Risk Evaluation and Microalbuminuria, $\mathrm{SD}=$ standard deviation, $\mathrm{BMI}=$ body mass index, $\mathrm{HDL}=$ high-density lipoprotein . *Unless stated otherwise. in blood pressure seen in the control group. In addition, there may have been a halo effect because patients in the intervention group and the control group shared family physicians. By witnessing effects on blood pressure control, a patient in the intervention group may have influenced his or her family physician's practice for managing hypertension in a control patient. In planning the study, we recognized that these effects could challenge the potential superior benefit of the treatment protocol but, because the care received would have the potential to improve overall health care delivery for the subjects and their community, we felt it to be a fair trade-off. The study was unblinded because it was unacceptable to conceal treatment in this population. There were insufficient family physicians in the region to randomize by practice. Because the sample was smaller than 60 in each group, a significant difference in the primary outcome measure may have been missed owing to a type II error. Finally, in addition to the effect of the visits by the home care nurse and education in both groups, confounding from interactions between subjects within a single reserve or subjects sharing a family physician may have affected the outcome of this study and should be explored in future investigations.

The DREAM 3 study was designed to evaluate the effectiveness of an algorithm of pharmocologic therapy on blood

Table 2: Blood pressure, urine albumin and hemoglobin $A_{1 c}$ values at baseline and at final visit

Group; mean (SD)

\begin{tabular}{|c|c|c|}
\hline \multirow{2}{*}{ Variable } & \multirow{2}{*}{ Intervention } & \multirow{2}{*}{ Control } \\
\hline & & \\
\hline \multicolumn{3}{|c|}{$\begin{array}{l}\text { Systolic blood pressure, } \\
\mathrm{mm} \mathrm{Hg}\end{array}$} \\
\hline Baseline & $149.7(10.5)$ & $150.5(19.1)$ \\
\hline Final visit & $125.7(16.6)$ & $133.5(18.1)$ \\
\hline Change over time & $-24.0(13.5) \dagger$ & $-17.0(18.6) \dagger$ \\
\hline$p$ value* $^{*}$ & $<0.001$ & $<0.001$ \\
\hline \multicolumn{3}{|c|}{$\begin{array}{l}\text { Diastolic blood pressure, } \\
\mathrm{mm} \mathrm{Hg}\end{array}$} \\
\hline Baseline & $87.1(8.4)$ & $84.2(11.1)$ \\
\hline Final visit & $75.5(12.7)$ & $77.4(11.3)$ \\
\hline Change over time & $-11.6(10.6) \ddagger$ & $-6.8(11.1) \ddagger$ \\
\hline$p$ value* & $<0.001$ & $<0.001$ \\
\hline \multicolumn{3}{|l|}{ Urine albumin, $\mathrm{mg} / \mathrm{L}$} \\
\hline Baseline & $157.8(507.0)$ & $164.8(446.0)$ \\
\hline Final visit & $115.5(391.0)$ & $124.9(369.0)$ \\
\hline Change over time & $-42.3(498.8) \dagger$ & $-40.9(364.9) \dagger$ \\
\hline$p$ value* & 0.57 & 0.71 \\
\hline \multicolumn{3}{|l|}{ Hemoglobin $A_{1 c}, \%$} \\
\hline Baseline & $7.7(1.8)$ & $7.7(1.8)$ \\
\hline Final visit & $7.8(2.1)$ & 7.7 (1.9) \\
\hline Change over time & $0.1(1.7) \dagger$ & $-0.0(1.3) \dagger$ \\
\hline$p$ value* & 0.68 & 0.87 \\
\hline
\end{tabular}

*For comparison within group over time.

$\dagger p>0.05$ (not significant) for comparison between groups over time. $\neq p=0.05$ for comparison between groups over time. 
Table 3: Urine albumin status at baseline and at final visit*

Status at final visit

\begin{tabular}{|c|c|c|c|c|c|c|}
\hline \multirow[b]{2}{*}{ Status at baseline } & \multicolumn{3}{|c|}{ Intervention group } & \multicolumn{3}{|c|}{ Control group } \\
\hline & $\begin{array}{l}\text { Normal urine } \\
\text { albumin }\end{array}$ & Microalbuminuria & $\begin{array}{c}\text { Overt } \\
\text { nephropathy }\end{array}$ & $\begin{array}{l}\text { Normal urine } \\
\text { albumin }\end{array}$ & Microalbuminuria & $\begin{array}{c}\text { Overt } \\
\text { nephropathy }\end{array}$ \\
\hline Normal urine albumin & 24 & 4 & 0 & 17 & 5 & 0 \\
\hline Microalbuminuria & 2 & 6 & 0 & 5 & 10 & 0 \\
\hline Overt nephropathy & 0 & 4 & 5 & 0 & 1 & 1 \\
\hline
\end{tabular}

*Urine albumin status for 84 patients (45 intervention, 39 control) who had urine albumin levels measured at both the baseline and final visits. Microalbuminuria is defined as urine albumin:creatinine ratio $>2.0 \mathrm{mg} / \mathrm{mmol}$ in women and $>2.8 \mathrm{mg} / \mathrm{mmol}$ in men. Overt nephropathy is defined as urine albumin:creatinine ratio $>28 \mathrm{mg} / \mathrm{mmol}$ in women and $>20 \mathrm{mg} / \mathrm{mmol}$ in $\mathrm{men}$.

pressure control implemented by a home care nurse in a community setting. High rates of blood pressure control in the community were achieved in both groups. New strategies such as that used in the DREAM 3 study and partnerships that involve family physicians, nurses and specialists are required if hypertension, a major reversible cardiovascular risk factor, is to be treated to target levels.

\section{This article has been peer reviewed.}

From the Sunnybrook and Women's College Health Sciences Centre (Tobe, Kiss, Szalai [deceased], Perkins), Toronto, Ont.; St. Paul's Hospital and University of Saskatchewan (Pylypchuk), Saskatoon, Sask.; and Battlefords Tribal Council Indian Health Services (Wentworth, Hartman, Ironstand, Hoppe), North Battleford, Sask.

Competing interests: None declared for Joan Wentworth, Alexander Kiss, John Paul Szalai, Nancy Perkins, Susan Hartman, Laurie Ironstand or Jacqueline Hoppe. Sheldon Tobe Sheldon Tobe has received funding for non-peerreviewed investigator-initiated research from Pfizer and Merck and for industry-supported contract research from Astra Zeneca, Pfizer, Ortho Biotec, Novartis, Bristol Myers Squibb, Sanofi, Amgen, Roche, Merck Frosst and Boehringer-Ingelheim, as well as speaker fees from Pfizer, Bristol Myers Squibb, Merck, Sanofi-Aventis, Amgen and Abbott. George Pylypchuk has a paid consultancy with Merck Frosst for the Expert MD education program for family physicians; he has also received educational grants and speaker fees from Merck Frosst, Biovail, Pfizer, BMS-Sanofi, Novartis, Amgen, Abbott and Boehringer Mannheim, and travel assistance to attend meetings from Biovail.

Contributors: Sheldon Tobe was responsible for the integrity and accuracy of the data analysis. All of the authors were responsible for the collection, management, and analysis and interpretation of the data and for the review and preparation of the manuscript.

Acknowledgement: Support for this study was provided by the Canadian Institutes of Health Research (CIHR), in partnership with Pfizer Canada. Neither the CIHR nor Pfizer Canada had a role in the design or conduct of the study or in the collection, management, analysis and interpretation of the data.

\section{REFERENCES}

I. Jones CA, Francis ME, Eberhardt MS, et al. Microalbuminuria in the US population: third National Health and Nutrition Examination Survey. Am J Kidney Dis 2002;39:445-59.

2. Dyck RF, Tan L. Rates and outcomes of diabetic end-stage renal disease among registered native people in Saskatchewan. CMAJ I994;I50(2):203-8.

3. Shah BR, Gunraj N, Hux JE. Markers of access to and quality of primary care for aboriginal people in Ontario, Canada. Am J Public Health 2003;93:798-802.

4. ALLHAT Officers and Coordinators for the ALLHAT Collaborative Research Group. The Antihypertensive and Lipid-Lowering Treatment to Prevent Heart Attack Trial. Major outcomes in high-risk hypertensive patients randomized to angiotensin-converting enzyme inhibitor or calcium channel blocker vs diuretic: the Antihyperten- sive and Lipid-Lowering Treatment to Prevent Heart Attack Trial (ALLHAT) [published erratum appears in JAMA 2003;289(2):I78]. JAMA 2002;288:298I-97.

5. Artinian NT, Washington OG, Templin TN. Effects of home telemonitoring and community-based monitoring on blood pressure control in urban African Americans: a pilot study. Heart Lung 200I;30:19I-9.

6. Pylypchuk G, Klassen J, Wentworth J, et al. Diabetes Risk Evaluation and Microalbuminuria (DREAM) in First Nations People of Saskatchewan [abstract]. Clin Invest Med I998;2I:S72.

7. Fava JL, Velicer WF, Prochaska JO. Applying the transtheoretical model to a representative sample of smokers. Addict Behav I995;20:189-203.

8. Meltzer S, Leiter L, Daneman D, et al. 1998 clinical practice guidelines for the management of diabetes in Canada. CMAJI998;159(8 Suppl):SI-29.

9. Fodor JG, Frohlich JJ, Genest JJG Jr, et al; Working Group on Hypercholesterolemia and Other Dyslipidemias. Recommendations for the management and treatment of dyslipidemia: report of the Working Group on Hypercholesterolemia and Other Dyslipidemias. CMAJ 2000;I62(I0):I44I-7.

Io. Management of dyslipidemia in adults with diabetes. American Diabetes Association. Diabetes Care I998;21:179-82.

II. Myers MG, Valdivieso MA. Use of an automated blood pressure recording device, the BpTRU, to reduce the "white coat effect" in routine practice. Am J Hyperten 2003;I6:494-7.

I2. McFarlane PA, Holden R, Harris SB, et al. Clinical Practice Guidelines Expert Committee. Canadian Diabetes Association 2003 clinical practice guidelines for the prevention and management of diabetes in Canada. Nephropathy. Can J Diabetes 2003;27:S66-7I.

I3. Hoy WE, Baker PR, Kelly AM, et al. Reducing premature death and renal failure in Australian aboriginals. A community-based cardiovascular and renal protective program. Med J Aust 2000;172:473-8.

I4. Hintze JL. PASS 6.o: Power analysis and sample size for Windows. Kaysville (UT): NCSS Software; 2000.

I5. Bakris GL, Williams M, Dworkin L, et al. Preserving renal function in adults with hypertension and diabetes: a consensus approach. National Kidney Foundation Hypertension and Diabetes Executive Committees Working Group. Am J Kidney Dis 2000;36:646-6I

I6. MacLean DR, Joffres MR, Tan MH, et al. Prevalence of cardiovascular risk factors in Canadians with diabetes mellitus. Adv Exp Med Biol 200I;498:373-80.

I7. Gaede P, Vedel P, Larsen N, Jensen GV, Parving HH, Pedersen O. Multifactorial intervention and cardiovascular disease in patients with type 2 diabetes. $N$ Engl Med 2003;348:383-93.

I8. Cost effectiveness analysis of improved blood pressure control in hypertensive patients with type 2 diabetes: UKPDS 40. UK Prospective Diabetes Study Group. BM I998;317:720-6.

I9. Hoy WE, Kondalsamy-Chennakesavan S, et al. A chronic disease outreach program for Aboriginal communities. Kidney Int SuppI 2005;98:S76-82.

20. Davidson MB. Effect of nurse-directed diabetes care in a minority population. Diabetes Care 2003;26:228I-7.

2I. Campbell NC, Ritchie LD, Thain J, Deans HG, Rawles JM, Squair JL. Secondary prevention in coronary heart disease: a randomised trial of nurse led clinics in primary care. Heart 1998;80:447-52.

22. Majumdar SR, Guirguis LM, Toth EL, Lewanczuk RZ, Lee TK, Johnson JA. Controlled trial of a multifaceted intervention for improving quality of care for rural patients with type 2 diabetes. Diabetes Care 2003;26:306I-6.

Correspondence to: Dr. Sheldon W. Tobe, Division of Nephrology, Sunnybrook and Women's College Health Sciences Centre, Rm. A240, 2075 Bayview Ave., Toronto ON M4N $3 M_{5}$; fax 4I6 480-6940; sheldon.tobe@sw.ca 